\title{
8
}
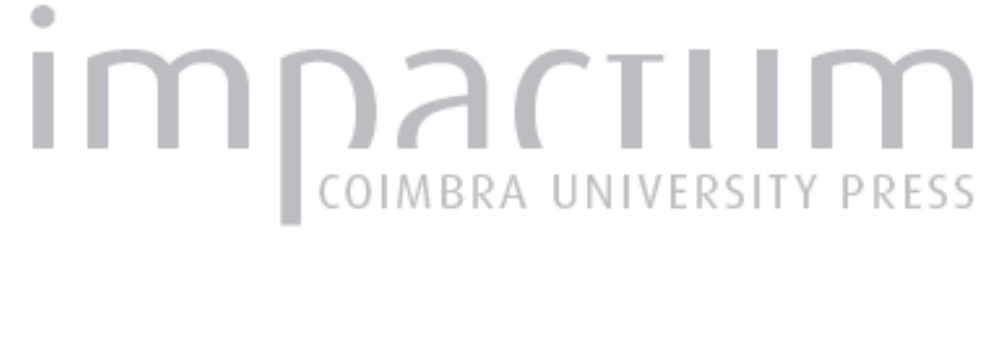

\section{Sur la reception de la philosophie Schelling en Europe et au Portugal (de la seconde moitie du XIXe siecle au debut du XXe)}

Autor(es): $\quad$ Ribeiro, Henrique Jales Publicado por: Faculdade de Letras da Universidade de Coimbra, Instituto de Estudos

URL

persistente:

DOI: $\quad$ DOI:http://dx.doi.org/10.14195/0872-0851_40_4

Accessed : $\quad$ 26-Apr-2023 13:53:02

A navegação consulta e descarregamento dos títulos inseridos nas Bibliotecas Digitais UC Digitalis, UC Pombalina e UC Impactum, pressupõem a aceitação plena e sem reservas dos Termos e Condições de Uso destas Bibliotecas Digitais, disponíveis em https://digitalis.uc.pt/pt-pt/termos.

Conforme exposto nos referidos Termos e Condições de Uso, o descarregamento de títulos de acesso restrito requer uma licença válida de autorização devendo o utilizador aceder ao(s) documento(s) a partir de um endereço de IP da instituição detentora da supramencionada licença.

Ao utilizador é apenas permitido o descarregamento para uso pessoal, pelo que o emprego do(s) título(s) descarregado(s) para outro fim, designadamente comercial, carece de autorização do respetivo autor ou editor da obra.

Na medida em que todas as obras da UC Digitalis se encontram protegidas pelo Código do Direito de Autor e Direitos Conexos e demais legislação aplicável, toda a cópia, parcial ou total, deste documento, nos casos em que é legalmente admitida, deverá conter ou fazer-se acompanhar por este aviso. 


\title{
SUR LA RECEPTION DE LA PHILOSOPHIE DE SCHELLING EN EUROPE ET AU PORTUGAL (DE LA SECONDE MOITIE DU XIXE SIECLE AU DEBUT DU XXE)
}

\author{
HENRIQUE JALES RIBEIRO*
}

Résumé: Ayant en vue l'étude de l'influence de la philosophie de Schelling pendant la seconde moitié du XIXe siècle - et en reprenant les suggestions de W. Wundt, É. Meyerson et E. Cassirer -, nous soutenons que cette époque, en général, se caractérise par la tentative de faire une synthèse entre les philosophies, opposées en plusieurs aspects, de Comte et de Hegel, dans le cadre de laquelle on revient, d'une façon plus ou moins éclectique, aux idées de l'auteur des Recherches philosophiques sur l'essence de la liberté humaine, et, en spécial, à sa philosophie de la nature. Nous étudions attentivement les traits principaux d'un tel contexte et de la philosophie de quelques uns des respectifs auteurs et tendances (comme F. Ravaisson et le spiritualisme en France, E. von Hartmann et l'évolutionnisme en Allemagne, A. de Quental, L. Coimbra, et leur réception de la philosophie européenne au Portugal).

Mot-Clefs: Comte, éclectisme, évolutionnisme, Hartmann, Hegel, philosophie au Portugal, positivisme, Ravaisson, Schelling, spiritualisme.

Resumo: Tendo em vista o estudo da influência da filosofia de Schelling durante a segunda metade do século XIX - e retomando as sugestões de W. Wundt, É. Meyerson e E. Cassirer -, defendemos que esta época, em geral, carateriza-se pela tentativa de fazer uma síntese entre as filosofias, opostas em vários

* Université de Coimbra (Département de Philosophie, Communication et Information). Cet article a été originellement élaboré dans le cadre du colloque «Action et histoire: la liberté humaine en question. Colloque international commémorant le bicentenaire des Recherches philosophiques sur l'essence de la liberté humaine, de F. W. J. Schelling» (Université de Coimbra, Faculté des Lettres, 19-21 novembre 2009). Je remercie aux organisateurs de la permission de le publier ici et pour la première fois. 
aspectos, de Comte e de Hegel, no quadro da qual se regressa, de uma forma mais ou menos eclética, às ideias do autor das Investigações filosóficas sobre a essência da liberdade humana. Estudamos atentivamente os traços principais de um tal contexto e da filosofia de alguns dos respetivos autores e tendências (como F. Ravaisson e o espiritualismo em França, E. von Hartmann e o evolucionismo na Alemanha, A. de Quental, Leonardo Coimbra, e a sua recepção da filosofia europeia em Portugal).

Palavras-chave: Comte, ecletismo, espiritualismo, evolucionismo, filosofia em Portugal, Hartmann, Hegel, positivismo, Ravaisson, Schelling.

\section{La réception}

À titre de préface, trois mots sur l'idée de réception. Le premier pour dire que, du point de vue de l'histoire de la philosophie, nous sommes tous des récepteurs; que nous sommes toujours sur le plan de la réception, même quand, ou surtout quand, nous essayons de comprendre la philosophie de Schelling dans son contexte historique et philosophique originel. On reçoit toujours, en projetant inexorablement dans un tel contexte des idées propres à notre contexte de réception; et l'idée que nous pourrions comprendre la pensée de Schelling elle-même indépendamment de notre contexte de réception (ou la pensée de n'importe quel philosophe indépendamment de ce genre de contexte), est une illusion dangereuse et profondément néfaste pour le travail de l'historien de la philosophie. C'est de ce point de vue méthodologique que je me place ici. Cela veut dire que, pour celui qui veut arriver à bien la connaître, la philosophie de Schelling doit être inévitablement projetée dans l'histoire de la philosophie jusqu'à nous, et que c'est dans ce parcours complexe qu'elle doit être vraiment appréciée.

Cela me conduit à ma seconde remarque. Il convient de souligner, surtout en ayant en vue le sujet de cet article, que le parcours auquel je fais allusion n'est pas une voie directe ou rectiligne: il n'est pas nécessaire, pendant la seconde moitié du XIXe siècle, pour présenter et développer quelques idées fondamentales apparentées à celles de Schelling, qu'on ait lu et réfléchi attentivement sur ses travaux; on peut être schellingien à cette époque-là sans avoir pris une connaissance directe des travaux de l'auteur des Recherches philosophiques sur l'essence de la liberté humaine. C'est principalement le cas des auteurs portugais (Antero de Quental et Leonardo Coimbra) dont je m'occuperai plus loin, et qui, pour la plupart, doivent être mis en rapport avec les idées de Schelling, non à travers une lecture directe de la langue allemande, 
ni même à travers les traductions faites de cet auteur vers le français, mais à travers les questions que la situation de la philosophie et de la science, à l'époque, les amenait inévitablement à poser et à discuter. Donc, ce n'est pas un problème de langue. Ce n'est pas non plus un problème de plus ou moins grande compétence intellectuelle, puisque, nous le verrons, la même chose arrive avec des philosophes français et anglais qui maîtrisaient parfaitement la langue et connaissaient bien la culture et la philosophie allemandes. De plus et d'une façon peut être plus importante et troublante pour nous aujourd'hui, on peut même parfois rejeter superficiellement quelques thèses de Schelling, en les confondant sous l'anathème, très commun à l'époque, de «hégélianisme»; et, cependant, les accepter et les développer dans un autre contexte. C'est un problème de pur questionnement philosophique qui dépend des caractéristiques très spéciales, sinon même uniques, de la situation de la philosophie et de la science à laquelle j'ai fait allusion. C'est ce genre de questionnement qui explique pourquoi je me permets de parler dans le titre de ma communication de «la réception de la philosophie de Schelling dans la seconde moitié du XIXe siècle».

Un troisième mot, un peu plus étendu mais final, sur ce contexte de réception. Comment discerner ses traits principaux? On ne peut le faire, au départ, que d'une façon tout à fait métahistorique et métaphilosophique, c'est-à-dire, qu'on est obligé de reconstruire un tel contexte. Cela veut dire que l'intérêt principal de mon étude doit être, non l'influence de la philosophie de Schelling sur un auteur en particulier (et on en connaît quelques-uns dans la seconde moitié du XIXe siècle, comme E. von Hartmann, en Allemagne, ou F. Ravaisson en France), ni sur plusieurs auteurs, comme on serait amené à le faire pour des raisons historiques et circonstancielles, mais, plutôt, la caractérisation des traits constitutionnels de sa réception. Au premier abord, on risque de parler moins de cette philosophie considérée en elle-même que de l'époque où elle intervient et prend sa place. Ceci est inévitable et ne doit pas nous faire désespérer en tant qu'historiens de la philosophie. Apparemment au moins, on sera très loin de la pensée de Schelling considérée historiquement: en ce qui concerne seulement l'histoire et la théorie de la science (et si l'on ignore l'histoire de la philosophie proprement dite), on devrait parler, par exemple, du débat entre les représentations mécanique et énergétique de la nature, spécialement au cours des deux dernières décennies du XIXe siècle, et de la façon dont ce débat a conduit à replacer et à renouveler, contre Hegel en particulier, la conception schellingienne de la philosophie de la nature et, en particulier, la conception d'une hiérarchie des degrés de l'être où la philosophie (la «philosophie transcendantale» selon Schelling) 
trouverait elle-même sa vraie place; ou encore, on devrait parler de la théorie de l'évolution des espèces telle que Darwin l'a introduite, et que Schelling n'a pas pu connaître en son temps, et de la façon dont cette théorie a complètement transformée, mais dans certains aspects en accord avec les vues de cet auteur, nos conceptions de la nature. On retrouve partout, à propos de ces sujets-là, les vues de Schelling, soit dans Les idées pour une philosophie de la nature, soit dans le Système de l'idéalisme transcendantal, ou, du moins, on trouve des vues apparentées à sa philosophie. Mais, étant données les caractéristiques spéciales de cette réception de la philosophie de Schelling, auxquelles je viens de faire allusion rapidement, est-ce vraiment de lui dont il est question? N'est-ce-pas plutôt d'un Hegel travesti et interprété selon les convenances philosophiques, transitoires, d'un temps où, sans croire à la philosophie de la nature de l'auteur berlinois, mais, quand même, sans pouvoir complètement s'en éloigner, on cherche un nouveau paradigme philosophique? Ou, plutôt, s'agit-il non seulement de Schelling et de Hegel, mais aussi, peut-être dans la confluence historique de ces deux grands philosophes, d'un Leibniz réinterprété et renouvelé d'après l'idée que, finalement, sa philosophie n'est pas complètement opposée à celle de Spinoza, son contemporain? C'est ce qui a été suggéré de façon brillante par Y. Belaval, il y a plus de trente ans, dans un texte que je me permets de reproduire ici en le mettant en rapport non seulement avec la réception de Leibniz, mais aussi avec celle de Schelling et de Hegel dans la seconde moitié du XIXe siècle:

«C'est dans la confusion qu'un auteur se diffuse. On le trouve partout et on ne peut le saisir nulle part. (...) Confusion, diffusion; mais aussi invention. Les erreurs de lectures, les méconnaissances ou les connaissances de seconde main modèlent peu à peu un nouvel auteur. L'histoire de la philosophie est une création continuée, une invention constante. On invente un leibnizianisme, et c'est ce leibnizianisme qui féconde les esprits. Quand on revient aux textes mêmes, on s'aperçoit du décalage. ${ }^{1}$

C'est précisément du contexte dont nous parle Belaval dans ces Études leibniziennes - lesquelles, apparemment du moins, n'ont pas eu de rivales jusqu'à aujourd'hui en ce qui concerne les études sur Schelling -, que j'aimerais pouvoir m'occuper dans ma communication à propos de ce grand philosophe.

${ }^{1}$ Y. Belaval, Études leibniziennes. De Leibniz à Hegel, Paris, Gallimard, 1976, p. 221. 


\section{Le contexte}

J'ai déjà suggéré qu'au lieu de chercher de façon plus au moins casuistique l'influence de Schelling pendant la seconde moitié du XIXe siècle, on doit reconstruire le contexte historique de la philosophie en général à l'époque où se produit la réception de sa pensée. La reconstruction résulte de quelques hypothèses «expérimentales» induites de la situation de la philosophie et de la science à cette période-là, et qui, à mon avis, peuvent être suffisamment confirmées ou corroborées chez la plupart des auteurs soit en Allemagne et en Angleterre, soit, surtout, en France. Elles peuvent l'être aussi, d'une façon peut-être plus remarquable, chez les philosophes portugais dont je vous ai parlé.

L'hypothèse fondamentale est l'idée d'une synthèse finale entre science et philosophie qui serait faite d'après les deux principaux systèmes philosophiques en Europe au début de la seconde moitié du XIXe siècle: celui de Comte ou du positivisme et celui de Hegel ou de l'idéalisme absolu. (Veuillez remarquer que je ne parle pas directement ici de Schelling et de sa philosophie. Son influence, bien que très importante, comme on le verra, est, pour moi, un cas spécifique de relecture et de réinterprétation de la philosophie de Hegel.) L'hypothèse a été suggérée pour la première fois par A. Vera, le premier grand traducteur de Hegel en français. ${ }^{2}$ L'historiographie d'Ernst Cassirer y souscrit aussi dans l'introduction de Le problème de la connaissance dans la philosophie et la science des temps modernes. De la mort de Hegel aux temps présents. ${ }^{3}$ Mais, avant Cassirer, E. Meyerson en France, dans son livre De l'explication dans les sciences, l'avait présentée en rappelant le témoignage, à ce sujet, de W. Wundt. ${ }^{4}$ En fait, on trouve cette même idée chez plusieurs philosophes de la seconde moitié du XIXe siècle. D'une certaine façon, le spiritualisme français se nourrit de celle-ci et Félix Ravaisson en dira quelque chose, indirectement, dans la conclusion de La philosophie en France au XIXe siècle. ${ }^{5}$ A. Fouillé la présente explicitement dans ses livres et surtout dans Le mouvement positiviste et la conception sociologique du monde. ${ }^{6}$ Mais d'autres, comme le portugais Antero de Quental dans ses Tendên-

${ }^{2}$ Voir «Introduction» à G. W. F. Hegel, La philosophie de l'esprit, vol. II, trad. par A. Vera, Paris, 1867.

3 Voir E. Cassirer, Das Erkenntnisproblem in der Philosophie und Wissenshaft der Neuren Zeit. Von Hegels Tod bis zur Gegenwart (1832-1932), Darmstadt, 1991.

${ }^{4}$ É. Meyerson, De l'explication dans les sciences, Paris, 1921, p. 123.

${ }^{5}$ F. Ravaisson, La philosophie en France au XIXe siècle, Paris, 1895, p. 249 ss.

${ }^{6}$ Voir A. Fouillé, Le mouvement positiviste et la conception sociologique du monde, Paris, 1900, p. 336 sq. 
cias gerais da filosofia na segunda metade do século XIX («Tendances générales de la philosophie dans la seconde moitié du XIXe siècle»), la soutiennent aussi. ${ }^{7}$

De quoi s'agit-il? L'idée est non seulement que les systèmes de Comte et de Hegel sont eux-mêmes des sortes de synthèses finales dans l'histoire de la philosophie occidentale, mais aussi (et principalement pour ceux qui acceptent l'idée comme programme de développement de la philosophie) que, si l'on tient compte des limites et des difficultés de chacun de ces systèmes et de la nouvelle situation de la science et de la philosophie (et ici, en ce qui concerne la science, je rappelle à nouveau l'importance de l'énergétisme et de la théorie de l'évolution), on doit les reconstruire en ayant en vue une synthèse créative et originale des deux. Bref: on souhaite faire la synthèse entre Comte et Hegel, ou entre les courants protagonistes de leurs conceptions philosophiques dans la seconde moitié du XIXe siècle. (Ravaisson, par exemple, parle d'une synthèse entre «matérialisme» et «idéalisme», mais d'autres parleront de «naturalisme» et de «spiritualisme», etc.). Cette ambition est à l'origine de l'éclectisme qui se répand sur toute la seconde moitié du XIXe siècle et qui, au contraire de ce que l'on pense d'habitude, est très loin d'être, en France, un simple épisode de la philosophie de V. Cousin (le grand introducteur des philosophies de Schelling et de Hegel dans ce pays). ${ }^{8}$ On est, pourrait-on dire, constitutionnellement éclectique; et même, dans certains cas, on fait l'éloge de l'éclectisme, comme il arrive en France avec Fouillé et, au Portugal, avec Quental et Coimbra. C'est pourquoi l'époque en question est celle où prolifèrent les soi-disant «philosophes mineurs». Si vous faites exception de Schopenhauer, Nietzsche et quelques autres, les philosophes en Europe pendant la seconde moitié du XIXe siècle, c'est-à-dire, pendant plus de cinquante ans, étaient, pour la plupart, des philosophes «mineurs».

C'est dans ce contexte complexe que se joue l'influence de Schelling ou, plutôt, de la réception de sa philosophie. Elle est habituellement indirecte; on évoque Schelling, on le mentionne ou on y fait allusion de façon élogieuse et en contraste avec ce qui se passe quant à Hegel, sans, toutefois, arriver à déterminer ou à spécifier, en détail, ses vues et, moins encore, le contraste dont je vous ai parlé. C'est le cas, par exemple, de

${ }^{7}$ A. de Quental, Tendências gerais da filosofia na segunda metade do século XIX. In: Obras completas de Antero de Quental, tome III, Lisboa, 1991, p. 168.

${ }^{8}$ Voir F. W. J. Schelling, Système de l'idéalisme transcendental, par M. de Schelling, suivi d'un jugement sur la philosophie de M. Victor Cousin, et sur l'état de la philosophie française, et la philosophie allemande, par le même auteur, trad. par P. Grimblot, Paris, 1842. Cf. D. Janicaud, «Victor Cousin et Ravaisson, lecteurs de Hegel». In: Les Études Philosophiques, 1984, p. 451 sq. 
La philosophie en France au XIXe siècle, de Ravaisson; ${ }^{9}$ mais on pourrait dire la même chose à propos des travaux de É. Boutroux ${ }^{10}$ ou de ceux de C. Renouvier. ${ }^{11}$ Mais laissez-moi être un peu plus explicite sur la caractérisation de la synthèse entre Comte et Hegel, entre positivisme et idéalisme, qui est le cadre vraiment central de la réception de la philosophie de Schelling pendant la seconde moitié du XIXème siècle, surtout en France et, par l'intermédiaire de ce pays, au Portugal.

Le concept clef pour comprendre ce cadre s'appelle «hiérarchie des sciences». On sait que ce concept joue un rôle fondamental soit dans le positivisme de Comte, où il apparaît en tant que tel («hiérarchie des sciences»), soit dans la philosophie de la nature de Hegel, où il intervient sous la forme de la distinction entre les trois degrés fondamentaux de la nature (le mécanique, le physique et l'organique) et les sciences respectives. La connexion épistémologique entre les deux concepts de la hiérarchie, celui de Comte et celui de Hegel, n'est ni complète ni parfaite. Il nous faut élargir la hiérarchie du Cours de philosophie positive aux domaines de ce que son auteur appelait la «synthèse subjective»; et la hiérarchie des degrés de la nature, de la Philosophie de la nature, à ceux de la philosophie de l'esprit. De toute façon, étant donné cet élargissement du concept de hiérarchie, on ne pouvait accepter, à l'époque, ni les présupposés philosophiques de la version de ce concept de la part du philosophe français, ni ceux de la version de l'allemand, pour des raisons différentes mais complémentaires. En ce qui concerne l'auteur du Cours, on sait qu'à l'époque la structure de la hiérarchie qui correspond aux sciences de sa «synthèse objective» était un problème controversé: on y mettait parfois et contre lui, la psychologie. Mais cela n'est pas important pour nous ici. Deux points sont fondamentaux: d'une part, on ne pouvait pas accepter l'idée comtienne selon laquelle la science ne peut jamais arriver à connaître les phénomènes eux-mêmes, mais seulement leurs rapports et, donc, on contestait clairement non seulement l'épistémologie de Comte et son refus de la métaphysique mais l'idée même de synthèse objective, qui traverse tout le Cours; d'autre part, on proposait ce que Fouillé appelait une «synthèse indivisiblement objective et subjective», c'est-à-dire- une synthèse selon laquelle la hiérarchie correspond à des degrés de l'être, à

${ }^{9}$ F. Ravaisson, op. cit., p. 33, 246, 281.

${ }^{10}$ Voir É. Boutroux, De la contingence des lois de la nature, Paris, 1895 (thèse de doctorat présentée en 1874); et idem, De l'idée de loi naturelle dans la science et la philosophie contemporaines, Paris, 1895.

11 Voir C. Renouvier, Philosophie analytique de l'histoire, vol. IV: Les idées, les religions, les systèmes, Paris, 1897; idem, La nouvelle monadologie, Paris, 1899; idem, Le personnalisme, suivi d'une étude sur la perception externe et la force, Paris, 1926. 
une échelle ontologique de celui-ci, et l'homme apparaît, tout près de la fin ou du sommet de échelle, comme, dira-t-il, «le naturel lui-même prenant conscience de sa vraie vie ou existence». ${ }^{12}$ Un tel «naturel» qui prend conscience de lui-même (veuillez remarquer une référence indirecte au concept schellingien d'inconscient) est l'homme interprété selon la théorie darwinienne de l'évolution, comme le fait E. von Hartmann. ${ }^{13}$ (Une petite parenthèse pour vous dire qu'il n'est pas surprenant que ces degrés de la nature et de l'homme soient compris d'après la théorie de l'évolution, et surtout d'après celle de Darwin, puisqu' il n'y avait à l'époque aucun sentiment d'incompatibilité entre les philosophies de Schelling et de Hegel et cette théorie, comme le montre, une fois de plus, la philosophie de Hartmann.) C'est dans les derniers degrés de l'être qui nous trouvons non seulement la conscience humaine mais aussi la liberté la plus essentielle et profonde, l'amour et Dieu. ${ }^{14} \mathrm{Ce}$ sont les domaines de l'art et de la religion; mais aussi, pour quelques-uns (comme les spiritualistes français), de ce qu'on appelait, sans apparemment penser à l'idéalisme absolu allemand, la «philosophie de l'esprit».

Maintenant, si l'on reprend dans son ensemble cette conception des «degrés de l'être» que je viens de résumer très rapidement, on pourrait remarquer que non seulement nous avons abandonné presque complètement le positivisme de Comte et ses développements plus au moins orthodoxes à l'époque (réalisme, matérialisme, naturalisme), mais aussi que, jusqu'à un certain point, nous sommes entrés, en apparence au moins, dans le territoire de l'idéalisme et de l'hégélianisme en particulier, ou, plutôt, dans un territoire partagé par Hegel et Schelling. L'idée centrale des deux philosophes allemands, si on veut éliminer tout ce qui est secondaire ou accessoire, est que les degrés de l'être sont des degrés de la nature et de l'homme, en tant que celui-ci appartient au règne de la nature, mais que, à un certain moment ou à une certaine étape (où commence la philosophie de l'esprit), il prend conscience de lui-même et de sa liberté et arrive par là, finalement, à la connaissance de Dieu ou de l'Absolu. D'une certaine manière, d'après cette idée fondamentale on retourne au panthéisme et à celui de Spinoza en particulier; et l'on connaît l'importance de la controverse entre théisme et panthéisme chez Schelling et Hegel et, à ce propos,

12 A. Fouillé, op. cit., p. 336.

${ }^{13}$ E. von Hartmann, Wahrheit und Irrthum im Darwinismus. Eine kritische Darstellung der organischen Entwickelungstheorie, Berlin, 1875 (trad. par G. Guéroult : Le darwinisme. Ce qu'il y a de vrai et de faux dans cette théorie, Paris, 1880); et idem, Philosophie des Unbewussten, Berlin, 1869 (trad. par D. Nolen : Philosophie de l'inconscient, Paris, 1877).

14 É. Boutroux, De la contingence des lois de la nature, Paris, 1895, p. 152 sq. 
les discussions sur la philosophie de l'auteur de l'Éthique. D'autre part, on revient clairement à la philosophie de la nature de Leibniz, en consonance avec les demandes du dynamisme en physique et de la théorie de l'énergie (ou l'énergétisme) en particulier. En effet, Leibniz était, à l'époque, une référence philosophique paradigmatique, principalement à cause du parallèle entre son concept métaphysique de force et le concept proprement scientifique et à cause de sa tentative de conciliation entre mécanisme et vitalisme dans la nature et chez l'homme. (Belaval, que j'ai cité plus haut, a écrit de belles pages à ce sujet.) Mais les théories de Leibniz elles-mêmes étaient reprises et d'une certaine façon renouvelées dans le cadre de l'idée d'une hiérarchie de degrés de la nature et de l'homme, bien que l'auteur de La Monadologie n'ait jamais vraiment pensé à une hiérarchie des monades qui correspondrait à une quelconque hiérarchie des sciences ou du savoir en général. C'est dans ce contexte historique, scientifique et philosophique complexe que l'on doit reprendre l'idée centrale que j'ai énoncée ci-dessus à propos de la réception des philosophies de Schelling et de Hegel pendant la seconde moitié du XIXe siècle. On sait qu'une telle idée a eu des expressions bien différentes chez les deux philosophes allemands dont on doit inévitablement s'occuper. Cependant, si on la considère dans la perspective de sa réception (et j'ai déjà suggéré que c'est peut-être la meilleure façon de la considérer aujourd'hui), de qui s'agit-il, en fait, dans cette nouvelle philosophie de la nature et de l'esprit qu'on trouve un peu partout éparpillée à l'époque? De Schelling ou de Hegel? Ou peut-être s'agirait-il des deux?

On doit remarquer, d'abord, qu'il ne s'agit pas, pour la plupart des philosophes, de reprendre directement ou indirectement Hegel, dont quelques travaux essentiels (comme la Phénoménologie de l'esprit, la Science de la logique et la Philosophie de la nature) avaient été traduits et commentés par A. Vera. ${ }^{15}$ Le présupposé général dans la seconde moitié du XIXe siècle (compte tenu de quelques exceptions, en Angleterre surtout) était que la philosophie de Hegel peut être identifiée, comme le dira Ravaisson et à quoi d'autres souscriront postérieurement, à un «mécanisme logique», c'est-à-dire, à une pure manipulation de concepts, ${ }^{16}$ et, donc, que cette philosophie dans son ensemble n'était plus vraiment que la Science de la logique travestie. ${ }^{17}$ En particulier, la thèse fondamentale de Hegel selon

15 G. W. F. Hegel, Logique, Paris, 1859; idem, La philosophie de la nature, Paris, 1863-1864; idem, La philosophie de l'esprit, Paris, 1867.

${ }^{16}$ F. Ravaisson, op. cit., p. 281.

17 Sur l'influence de Hegel en France, voir B. Bruce . «Limiting Reason's Empire: The Early Reception of Hegel in France». In: Journal of the History of Philosophy, 
laquelle la nature est l'«Idée dans son être-autre», ou que "dans la nature l'esprit est hors de lui-même» ou extériorisé, ne pouvait pas être acceptée du tout. Elle était prise comme une dévaluation complète non seulement des sciences de la nature, à une époque où celles-ci devenaient de plus en plus importantes, mais de l'idée de nature elle-même. Mais, comme je l'ai déjà suggéré, de cette thèse-là la plupart des auteurs déduisaient précipitamment, et parfois en contradiction avec les résultats de leurs propres travaux, le caractère insoutenable de la philosophie de la nature hégélienne et de sa dissociation entre nature et esprit. C'est une thèse qui, d'après quelques commentateurs, est restée presque intouchable jusqu'à nos jours. ${ }^{18}$

Par contre, la philosophie de l'identité de Schelling aurait montré non seulement l'unité entre la nature et l'esprit, mais aussi comment le même principe organisateur qui a créé inconsciemment la nature et la série graduelle de ses produits, l'Esprit ou l'Absolu proprement dit, peut devenir conscient de lui-même chez l'homme et se présenter finalement comme la seule vraie force productrice. Voilà l'idée générale contre Hegel que l'on trouve partagée et acceptée, d'une façon générale, par ceux qui s'intéressaient à la philosophie de la nature pendant la seconde moitié du XIXe siècle. Il faut l'examiner, parce que c'est justement dans quelques détails que l'on peut évaluer l'influence réelle de l'auteur du Système, et essayer de comprendre la confusion qui existait à l'époque entre Schelling et Hegel sous l'épithète d' «hégélianisme». On trouve un dédoublement pareil à celui du principe de Schelling dans la philosophie de Hegel: c'est toujours la même Idée qui s'extériorise dans la nature et qui revient à soi-même dans une étape supérieure. ${ }^{19}$ Mais que le principe soit conçu à travers les concepts de force, activité, liberté, au contraire de ce qui arrivait dans la philosophie de Hegel, où, en apparence au moins, il est réduit au jeu dialectique de l'Idée (à un «mécanisme logique», comme diront Ravaisson, Boutroux; et d'autres), aux fondements conceptuels du développement de celle-ci, cela faisait toute la différence. On pourrait dire la même chose à propos de l'auto-conscience de ce principe dans la volonté humaine, qui est un des thèmes principaux des Recherches. On suivait Schelling, en refusant clairement Hegel et sa philosophie de la nature, dès le moment où les concepts de sa philosophie, y compris le concept même d'Esprit ou d'Absolu, pouvaient être compris selon de présupposés essentiellement anthropologiques et psychologiques, c'est-

31/2 (1993), p. 259-275; et V. Mudimbe, \& A. Bohm, «Hegel's Reception in France». In:

http://web.ics.purdue.edu/ smith132/French_Philosophy/Fa94/hegel.pdf

18 Voir E. Renault, La naturalisation de la dialectique, Paris, 2001, p. 7-13.

19 Voir idem, ibidem, p. 54 sq. 
-à-dire, mis en correspondance avec l'expérience humaine; on le sous-estimait ou même on l'ignorait, en le confondant avec Hegel et l'«hégélianisme», quand il entrait dans le domaine de la philosophie transcendantale proprement dite.

Cette réception de Schelling était en accord avec les données, à l'époque, de la théorie de la science, parce que ce que celle-ci montrait, dès la dynamique et les principes de l'énergie, en physique, jusqu'à la biologie et à la théorie de l'évolution en particulier, c'était l'importance fondamentale des concepts de force et d'activité. Elle était aussi en accord avec le retour à la philosophie de Leibniz et à sa théorie de la substance en particulier, que l'on croyait être capable, en un sens, d'expliquer certains domaines scientifiques, comme la dynamique, mais aussi être complètement inapte à s'appliquer à l'idée d'une hiérarchie de degrés de la nature, qui était justement l'un des points principaux de l'intérêt pour la philosophie de la nature de Schelling, au moins quand celle-ci était mise en contraste avec celle de Hegel. D'ailleurs, on sait que Schelling luimême a insisté à plusieurs endroits précisément sur ce rapport entre ses propres idées et celles de l'auteur de La Monadologie. Et, en effet, dans la seconde moitié du XIXe siècle on trouve un peu partout l'association entre les idées de force, d'activité, d'énergie et l'idée d'un principe qui serait sous-jacent au développement ou à l'évolution des degrés de la nature, soit, en France, dans le spiritualisme de Boutroux et d'autres, ou dans le criticisme de C. Renouvier et la philosophie des idées-forces de A. Fouillé, ${ }^{20}$ soit, en Angleterre, chez H. Spencer, ${ }^{21}$ soit, en Allemagne, chez W. Wundt ${ }^{22}$ et, comme nous l'avons déjà signalé jusqu'ici, dans la philosophie de E. von Hartmann.

Mais, d'autre part, l'idée que l'Esprit puisse être inconscient dans la nature, ou que celle-ci seulement devienne consciente de soi chez l'homme, au sommet de la série graduelle de ses produits, n'était pas moins attractive. On doit remarquer que l'on peut trouver cette même thèse, avec d'autres présupposés, dans la philosophie de la nature de Hegel. Mais sur ce point, à nouveau, à l'époque, l'on réduisait l'idée hégélienne de l'inconscient dans la nature à ses fondements dialectiques; elle ferait partie d'un développement purement idéal de la raison, et non d'un développement réel de la nature. ${ }^{23} \mathrm{Au}$ contraire, le concept schellingien de la nature comme organisme, c'est-à-dire comme un grand être

${ }^{20}$ Cf. George-Lespinasse Fonsegrive, De Taine à Péguy. L'évolution des idées dans la France contemporaine, Paris, 1916, p. 97 sq.

${ }^{21}$ Cf. H. Spencer, First Principles, London, 1862.

22 Voir W. Wundt, System der Philosophie, Leipzig, 1889, 2 vol.

${ }^{23}$ É. Boutroux, Études d'histoire de la philosophie allemande, Paris, 1926, p. 95 sq. 
vivant où tout se trouve parfaitement harmonisé, assurerait au concept d'inconscient de cet auteur une légitimité complètement différente dès le départ. Ce concept pourrait être mis en connexion non seulement avec la philosophie de la volonté, de Schopenhauer, mais aussi et principalement avec la théorie de l'évolution; de Darwin et d'autres. C'est ce que Hartmann a soutenu ${ }^{24}$ et, après lui, beaucoup d'autres philosophes, y compris les portugais Antero de Quental et Leonardo Coimbra. ${ }^{25}$ Le point essentiel, de ce point de vue, était que, comme pour l'auteur des Idées, du Système et des Recherches, il n'y avait pas deux natures différentes, l'organique et la non-organique, mais que dans celle-ci était déjà présent le même principe unitaire d'évolution qui est sous-jacent au développement de la série des degrés jusqu'à son auto-conscience chez l'homme.

Comme dans la philosophie de Schelling, cette thèse conduisait à renouveler le vieux débat entre mécanisme et vitalisme, mais maintenant, c'est-à-dire- dans les dernières décennies du XIXe siècle, cela arrivait pour des raisons beaucoup plus pressantes, qui concernaient la critique de l'image mécaniste du monde, prépondérante à l'époque. C'était sur le terrain épistémologique de la justification des séries de phénomènes propres à la hiérarchie de degrés de l'être que ce débat était reconduit, en montrant comment avec les êtres organisés et la vie la nature apparaît comme une finalité voulue et, avec l'homme, comme action libre, et, donc, comment la dite opposition peut être complètement surpassée dans le cadre même de la hiérarchie. Le spiritualisme français a développé ce genre d'approche, que l'on peut trouver déjà dans les travaux de Schelling dès les Idées. La contradiction transcendantale, développée par le Système, entre une nature qui serait simultanément mécanique et téléologique selon le dédoublement de l'activité de 1'Esprit ou de l'Absolu (d'une part inconscient et de l'autre conscient), n'intéressait pas du tout à l'époque, étant donné le refus d'une approche de ce type, auquel j'ai déjà fait allusion, c'est-à-dire le refus d'une approche où l'idée d'un «savoir du savoir» devient fondamentale. On allait de la nature vers l'esprit, sans jamais vouloir effectuer le parcours à l'envers (et, donc, construire la nature) à cause de son «hégélianisme» et, en particulier, de la tentation de faire de l'esprit une entité transcendante. L'idée fondamentale était que l'apparition de la liberté dans la hiérarchie de degrés de l'être avait ses fondements dans l'évolution jusqu'à l'homme, par degrés, de la nature elle-même. Compte tenu du refus de la perspective transcendantale en général, c'était plutôt dans cette

24 E. von Hartmann, Schellings philosophisches System, Leipzig, 1897.

25 Voir A. de Quental, op. cit., p. 128; et L. Coimbra, O pensamento filosófico de Antero de Quental (1921). In: Obras completas, tome IV, Lisboa, 2007, p. 329 sq. 
évolution que dans une quelconque activité productive et indépendante de l'Esprit ou de l'Absolu (ou de ses différentes versions à l'époque) que l'on allait chercher les raisons de la transition par degrés dans la hiérarchie. ${ }^{26}$ Mais, sur ce point, ce n'était pas seulement à Schelling que l'on pensait, mais surtout à un Leibniz corrigé ou, plutôt, à un Leibniz relu d'après quelques idées soit de Hegel soit, principalement, de l'auteur des Idées et du Système.

S'il est vrai que les idées de force et d'activité sont essentielles dans la théorie leibnizienne de la substance, comme elles l'étaient aussi pour la philosophie de la science pendant la seconde moitié du XIXe siècle; s'il est vrai, de plus, que cette théorie permettait de repenser, depuis l'idéalisme critique de Kant, le rapport entre mécanisme et vitalisme d'une façon complètement nouvelle, en présentant ses fondations métaphysiques dans la substance même; le fait est que, comme je l'ai déjà dit, on ne trouve pas, chez Leibniz, l'idée d'une hiérarchie de degrés de l'être qui puisse être mise en correspondance avec l'idée d'une hiérarchie du savoir ou des sciences, comme il arrive dans l'idéalisme de Schelling et de Hegel; et que sa solution finale, théologique, pour la controverse mécanisme versus vitalisme, c'est-àdire, l'harmonie préétablie, ne pouvait être du tout acceptée par une philosophie qui prétendait, avant toutes choses, être d'accord avec les derniers résultats des sciences naturelles. Dans la confluence de tous ces facteurs, la synthèse cherchée à l'époque passait, en général, d'une part, par un retour aux idées leibniziennes de force et d'activité comme principes dynamiques de la transition entre les degrés de la hiérarchie - ce qui était parfaitement en accord avec la philosophie de Schelling - et parfois même, explicitement, par un retour au concept de monade comme source substantielle de cette hiérarchie (comme il arrivait avec Charles Renouvier en France et Leonardo Coimbra au Portugal), et, d'autre part, par un refus de justifier la transition elle-même (épistémologiquement parlant) avec les outils du «mécanisme logique» hégélien, pour les mêmes raisons que j'ai déjà suggérées auparavant. Si l'on met de côté la perspective transcendantale de Schelling, les concepts que celui-ci avait présentés à propos de cette transition étaient beaucoup plus proches de l'ambiance intellectuelle de l'époque, et, en particulier, d'une lecture tendanciellement anthropologique de l'esprit et de son rapport avec la nature, que ceux que Hegel avait fournis dans ses travaux.

26 Voir É. Boutroux, La nature et l'esprit, Paris, 1926. 


\section{Développements au Portugal}

On peut rencontrer tous les aspects fondamentaux du contexte que je viens de reconstruire chez les philosophes portugais Antero de Quental (1842-1891) et Leonardo Coimbra (1883-1936). En général, ils ne parlent pas directement de la philosophie de Schelling dans leurs œuvres. Tous deux la connaissaient, apparemment, à travers la traduction de Grimblot du Système ${ }^{27}$ et le dernier, un peu plus tard, également à travers la traduction des Recherches par G. Politzer. ${ }^{28}$ Mais, sans doute, la connaissaient-ils indirectement par l'entremise de sa réception soit chez Cousin, Ravaisson et le spiritualisme français de la seconde moitié du XIXe siècle, soit chez des auteurs allemands influencés par elle et dont les œuvres avaient été traduites en français (comme c'est le cas de Hartmann). Plutôt que de parler explicitement de Schelling, ils l'évoquent, ils font allusion à lui (comme dirait Y. Belaval) de deux façons principales: d'abord, pour le critiquer et le contester d'une façon générale, sous prétexte qu'il appartiendrait à l' «hégélianisme» allemand de façon générale, c'est-à-dire, à une philosophie «aprioriste», divorcée de l'expérience et, en particulier, de celle des sciences de la nature; mais aussi et surtout, contradictoirement, pour s'identifier avec les résultats de sa philosophie de la nature quand celle-ci, une fois dissociée autant que possible de ses éléments transcendantaux, était lue selon la réinterprétation de Comte et de Leibniz dont je vous ai parlé auparavant, et mise en contraste avec celle de Hegel. Le résultat final était l'éclectisme. C'est ainsi qu'on peut trouver chez Antero de Quental, dans son explication du principe qui serait sous-jacent au passage par degrés de la hiérarchie des sciences, un appel pour les idées de «force», d'«immanence», de «développement», en tant qu' idées qui représenteraient la synthèse finale de la philosophie à la fin du XIXe siècle et qui orienteraient toute l'évolution de la philosophie de la nature dans le futur. ${ }^{29}$ Toutefois, chez ce philosophe portugais, nous restons toujours dans le parcours de la nature vers l'esprit, et non à rebours, c'est-à-dire dans le parcours que le spiritualisme français de Ravaisson, de Boutroux et d'autres avait déjà parcouru et qui, à la fin, signifie la négation du transcendantal de Schelling et du concept d'un «savoir du savoir» qui caractérise l'idéalisme absolu allemand en général.

L'exception à cette conclusion fondamentale, à laquelle je suis déjà arrivé en parlant de la réception de Schelling dans la philosophie euro-

${ }^{27}$ Cf. note 8 supra.

${ }^{28}$ F. W. J. Schelling, Recherches philosophiques sur l'essence de la liberté humaine et sur les problèmes qui s'y rattachent, Paris, 1926.

${ }^{29}$ A. de Quental, op. cit., p. 121 sq. 
péenne de la seconde moitié du XIXe siècle, est constituée par Leonardo Coimbra, l'autre philosophe portugais qui m'intéresse particulièrement ici, et qui, comme le premier, a été toujours pratiquement inconnu en Europe. En accord avec Antero de Quental, son prédécesseur, il constate, dans le premier livre qu'il a publié, en 1912, et dont le titre pourrait être traduit par Le créationnisme, les défauts de Hegel dans sa philosophie de la nature et l'incompétence de celle-ci pour rendre compte des nouvelles données des sciences. Mais il n'accepte pas le refus d'une approche transcendantale en philosophie de la part du spiritualisme français, avec sa distinction entre «immanence» et «transcendance» (qui était un moyen subtil d'éluder la question de la fondation de la philosophie et de la connaissance de façon générale), et il développe, indépendamment de toute influence directe de Schelling, l'idée d'un «savoir du savoir» qui aurait pour sujet l'étude du dédoublement de l'activité de l'esprit en tant que celui-ci serait inconscient dans la nature, et simultanément conscient, productif et libre dans la volonté humaine..$^{30}$ L'esprit était pour Leonardo Coimbra, comme pour le philosophe allemand, essentiellement création et liberté, qui se manifestent non seulement dans ce dédoublement mais aussi dans le savoir même que l'homme a de lui. C'est en ce sens que, selon Coimbra, le créationnisme, c'est-à-dire sa propre philosophie, pouvait être conçue comme un «néo-hégélianisme», et que celui-ci «est la philosophie la plus proche d'une philosophie de la liberté». ${ }^{31}$ Il revient donc, sans jamais mentionner ou parler explicitement de Schelling (et peut-être sans l'avoir lu attentivement), à la thèse de l'identité entre philosophie de la nature et philosophie de l'esprit, au moins quand l'une et l'autre étaient réinterprétées à la lumière des derniers résultats de la science et de la philosophie au début du XXe siècle.

\section{En guise de conclusion}

Pendant la seconde moitié du XIXe siècle et après la défaite apparente tant du positivisme de Comte que de l'idéalisme de Hegel, on rencontre Schelling, on arrive à lui et, en particulier, à sa thèse de l'identité, dans le cadre de la recherche d'une nouvelle synthèse entre science et philosophie qui serait la synthèse finale de la pensée philosophique moderne (Leibniz, Spinoza, Kant) et occidentale de façon générale. En dépit de tout, ce schellingianisme était, donc, une forme d'éclectisme. Mais il s'agissait

30 Voir L. Coimbra, O criacionismo (esboço de um sistema filosófico). In: Obras completas (1903-1912), tome II, Lisboa, 2004.

31 Voir idem, «A filosofia da liberdade». In: ibidem, tome I, p. 295. 
aussi, à la fin, d'un demi-schellingianisme: comme F. Ravaisson et les philosophes spiritualistes en France, on suivait Schelling - en pensant suivre simultanément Leibniz - quand il allait de la nature vers l'esprit et appelait aux idées de force, d'activité, de liberté, pour expliquer cette transition fondamentale, ou quand il concevait l'activité productive de l'esprit selon ce genre de prédicats métaphysiques; on le rejetait, sauf quelques honorables exceptions, au moment où il entrait dans sa philosophie transcendantale proprement dite et voulait, non seulement expliquer, mais encore construire spéculativement la nature.

\section{BIBLIOGRAPHIE}

BELAVAL, Yvon, Études leibniziennes. De Leibniz à Hegel, Paris, 1976.

BOUTROUX, Émile, De la contingence des lois de la nature, Paris, 1895.

Idem, De l'idée de loi naturelle dans la science et la philosophie contemporaines, Paris, 1895.

Idem, Études d'histoire de la philosophie allemande, Paris, 1926.

Idem, La nature et l'esprit, Paris, 1926.

BRUCE, B., «Limiting Reason's Empire: The Early Reception of Hegel in France». In : Journal of the History of Philosophy, 31/2 (1993), p. 259-275.

CASSIRER, Ernst, Das Erkenntnisproblem in der Philosophie und Wissenshaft der Neuren Zeit. Von Hegels Tod bis zur Gegenwart (1832-1932), Darmstadt, 1991.

COIMBRA, Leonardo, O criacionismo (esboço de um sistema filosófico). In: Obras completas (1903-1912), vol. I, tome II, Lisboa, 2004.

Idem, «A filosofia da liberdade». In: ibidem, vol. I, tome I, Lisboa, 2004.

Idem, O pensamento filosófico de Antero de Quental (1921). In: Obras completas, tome IV, Lisboa, 2007.

FOUILLÉ, Alfred, Le mouvement positiviste et la conception sociologique du monde, Paris, 1900.

HARTMANN, Eduard von, Philosophie des Unbewussten, Berlin, 1869.

Idem, Wahrheit und Irrthum im Darwinismus. Eine kritische Darstellung der organischen Entwickelungstheorie, Berlin, 1875.

Idem, Philosophie de l'inconscient, trad. par D. Nolen, Paris, 1877.

Idem, Le darwinisme. Ce qu'il y a de vrai et de faux dans cette théorie, trad. par G. Guéroult, Paris, 1880.

Idem, Schellings philosophisches System, Leipzig, 1897. 
HEGEL, Georg Wilhelm Friedrich, Logique de Hegel, trad. par Augusto Vera, Paris, 1859.

Idem, Philosophie de la nature, trad. par Augusto Vera, Paris, 1863-1866.

Idem, La philosophie de l'esprit, trad. par Augusto Vera, Paris, 1867.

JANICAUD, Dominique, «Victor Cousin et Ravaisson, lecteurs de Hegel». In: Les Études Philosophiques (1984), p. 451-466.

MEYERSON, Émile, De l'explication dans les sciences, Paris, 1921.

MUDIMBE, V., \& Bohm, A., «Hegel's Reception in France». In : http://web.ics. purdue.edu/ smith132/French_Philosophy/Fa94/hegel.pdf

FONSEGRIVE, George-Lespinasse, De Taine à Péguy: L'évolution des idées dans la France contemporaine, Paris, 1916.

QUENTAL, Antero de, Tendências gerais da filosofia na segunda metade do século XIX. In: Obras completas de Antero de Quental, tomo III, Lisboa, 1991.

RAVAISSON, Félix, La philosophie en France au XIXe siècle, Paris, 1895.

RENAULT, Emmanuel, La naturalisation de la dialectique, Paris, 2001.

RENOUVIER, Charles, Philosophie analytique de l'histoire, vol. IV: Les idées, les religions, les systèmes, Paris, 1897.

Idem, La nouvelle monadologie, Paris, 1899.

Idem, Le personnalisme, suivi d'une étude sur la perception externe et la force, Paris, 1926.

SCHELLING, Friedrich Wilhelm Joseph von, Système de l'idéalisme transcendental, par M. de Schelling, suivi d'un jugement sur la philosophie de M. Victor Cousin, et sur l'état de la philosophie française, et la philosophie allemande, trad. par P. Grimblot, Paris, 1842.

Idem, Recherches philosophiques sur l'essence de la liberté humaine et sur les problèmes qui s'y rattachent, trad. par Georges Politzer, Paris, 1926.

SPENCER, Herbert, First Principles, London, 1862.

WUNDT, Wilhelm, System der Philosophie, Leipzig, 1889. 
